\title{
A Note on the First Integrals of Vector Fields with Integrating Factors and Normalizers
}

\author{
Jaume LLIBRE ${ }^{\dagger}$ and Daniel PERALTA-SALAS ${ }^{\ddagger}$ \\ $\dagger$ Departament de Matemàtiques, Universitat Autònoma de Barcelona, \\ 08193 Bellaterra, Barcelona, Catalonia, Spain \\ E-mail: jllibre@mat.uab.cat \\ URL: http://www.gsd.uab.es/personal/jllibre \\ ¥ Instituto de Ciencias Matemáticas, Consejo Superior de Investigaciones Científicas, \\ C/ Nicolás Cabrera 13-15, 28049 Madrid, Spain \\ E-mail: dperalta@icmat.es \\ URL: http://www.icmat.es/dperalta
}

Received February 16, 2012, in final form June 12, 2012; Published online June 14, 2012

http://dx.doi.org/10.3842/SIGMA.2012.035

\begin{abstract}
We prove a sufficient condition for the existence of explicit first integrals for vector fields which admit an integrating factor. This theorem recovers and extends previous results in the literature on the integrability of vector fields which are volume preserving and possess nontrivial normalizers. Our approach is geometric and coordinate-free and hence it works on any smooth orientable manifold.
\end{abstract}

Key words: first integral; vector field; integrating factor; normalizer

2010 Mathematics Subject Classification: 34C05; 34A34; 34C14

\section{Introduction and statement of the main result}

Let $M$ be a smooth orientable manifold of dimension $n$ endowed with a volume form $\Omega$. We denote by $C^{\infty}(M)$ and $\mathfrak{X}^{\infty}(M)$ the spaces of smooth real-valued functions and vector fields on $M$ respectively. We shall only work in the smooth $\left(C^{\infty}\right)$ category, although most of our results can be easily adapted to lower regularity.

This letter is focused on the integrability properties of vector fields. More precisely, we are interested in proving some sufficient conditions which imply the existence of explicit first integrals (i.e. computable). We recall that a function $H \in C^{\infty}(U)$, where $U \subseteq M$ is an open subset of $M$, is a first integral of the vector field $X$ in $U$ if $L_{X}(H)=0$, which implies that $H$ is constant along the integral curves of $X$. As usual, $L_{X}$ denotes the Lie derivative with respect to the vector field $X$. We recall that an equivalent way of writing the first integral condition is $L_{X}(H)=i_{X}(d H)=X(H)=0$, where $d$ is the exterior derivative and $i$ denotes de contraction operator. In this paper we are interested in global or semi-global first integrals in the sense that the open set $U \subseteq M$ where the first integral is well defined is known a priori.

The importance of the existence of a non-constant first integral lies in the fact that the trajectories of the vector field $X$ leave invariant the level sets of the function $H$, and hence this is a strong constraint on the dynamical behavior of the vector field (e.g. it prevents from the existence of ergodic trajectories on $M$ ). Unfortunately, it is generally very difficult to compute a first integral (whose existence is, in fact, a rather non-generic phenomenon). This is the reason why it is necessary to introduce some auxiliary objects to study the integrability properties of a vector field. 
There are two important tools which can be used to analyze the existence of first integrals: integrating factors and normalizers. We say that a function $f \in C^{\infty}(M)$ is an integrating factor of the vector field $X$ (with respect to a volume form $\Omega$ ) if $\operatorname{div}_{\Omega}(f X)=0$. Recall that the divergence of a vector field $X$ with respect to a volume form $\Omega, \operatorname{div}_{\Omega}(X)$, is defined by $L_{X}(\Omega)=\operatorname{div}_{\Omega}(X) \Omega$. A vector field $Y \in \mathfrak{X}^{\infty}(M)$ is a normalizer of $X$ if $[X, Y]=\lambda X$ for some function $\lambda \in C^{\infty}(M)$ (the notion of normalizer was introduced by Lie and Engel, see e.g. the recent translation [14, Theorem 20, Chapter 8]). Remember that $[\cdot, \cdot]$ stands for the Lie bracket, which is defined as $[X, Y]=L_{X}(Y)$. Normalizers are also called orbital symmetries, and in the case that $[X, Y]=0$, it is usually said that the vector field $Y$ is a centralizer (or symmetry) of $X$.

There is an abundant literature on the connection between first integrals, integrating factors and normalizers. The fact that the existence of normalizers gives rise to computable first integrals in terms of quadratures is well known since Lie's works, see e.g. the monographs on the subject by Olver [16] and Bluman-Anco [3] or the comprehensive geometric account of reduction and integrability by Sherring and Prince [20]. Some recent results on the relationship between symmetries and first integrals can be consulted in $[4,13,17,19]$ and references therein. On the other hand, the existence of integrating factors is not generally enough to ensure the existence of first integrals, except for the case that $M$ has dimension 2 [8, Chapter 2.11]. In the case that $M=\mathbb{R}^{n}$ and the vector field $X$ is polynomial, some algebraic algorithms have been proposed to compute polynomial integrating factors and elementary first integrals [18, 21]. For volume-preserving vector fields, i.e. $\operatorname{div}_{\Omega}(X)=0$ (a particular case of vector fields admitting an integrating factor) which have volume-preserving normalizers, there are several procedures to compute explicit first integrals using quadratures $[9,11,15]$. We also note that integrating factors are also directly related to normalizers, and in fact can be obtained from them under suitable hypotheses, see e.g. [5].

In this letter we follow a totally different approach, which combines the existence of integrating factors with a suitable extension of the notion of normalizer (see $(i)$ below). The main difference with the approaches explained in the previous paragraph is that we provide an explicit formula for the first integral of the vector field in terms of the normalizer and the integrating factor, and there is no need of quadratures, reduction of order or algebraic manipulations to get this first integral. Previous results in this direction were obtained in $[6,7,10]$ for volume preserving vector fields with centralizers (see Section 3 for more details). Let us now state the main theorem of this letter.

Theorem 1.1. Let $X$ be a smooth vector field on a manifold $M$. Assume that the following three conditions hold:

(i) There exists a vector field $Y \in \mathfrak{X}^{\infty}(M)$ and two functions $\lambda, \mu \in C^{\infty}(M)$ such that $[X, Y]=$ $\lambda X+\mu Y$.

(ii) $X$ admits an integrating factor $f \in C^{\infty}(M)$.

(iii) The function $f \mu$ is an integrating factor of $Y$.

Then the function

$$
H:=\frac{L_{Y} f}{f}+\operatorname{div}_{\Omega}(Y)-\lambda
$$

is a smooth first integral of the vector field $X$ in the open set $U:=M \backslash Z_{f}$, where $Z_{f}:=\{p \in$ $M: f(p)=0\}$.

Remark 1.1. The theorem holds true if $X, Y$ and $f$ are of class $C^{2}$; in this case the first integral $H$ is of class $C^{1}$. On the other hand, if the zero set $Z_{f}$ has empty interior the first 
integral $H$ is defined in an open and dense subset of $M$. This is the case, e.g. if the integrating factor $f$ is analytic $\left(C^{\omega}\right)$.

Let us briefly explain the geometric meaning of the first integral derived in Theorem 1.1, cf. equation (1.1). We are very grateful to one of the referees for pointing out to us the argument that we sketch below. It is obvious that the function $g:=H f$ is a second integrating factor of the vector field $X$; conversely, if $X$ admits two integrating factors $f$ and $g$ it is ready to check that the function $I:=g / f$ is a first integral of $X$, but it does not need to be the same as the first integral $H$ computed in Theorem 1.1 (see e.g. Example 2.1 in Section 2). The point is that under the assumptions of Theorem 1.1 it is possible to compute a second integrating factor $g$ using the vector field $Y$ and the integrating factor $f$, such that the function $g / f$ is precisely the first integral $H$ given by equation (1.1). For example, in the particular case that $Y$ is a symmetry of $X$ (i.e. $[X, Y]=0$ ), then $g:=L_{Y} f$ is a second integrating factor of $X$, thus implying that $H:=\frac{L_{Y} f}{f}$ is a first integral of the vector field $X$. In the general case, one can look for a second integrating factor of the form $g:=L_{Y} f+\hat{g}$, and Theorem 1.1 provides some sufficient conditions to compute the unknown function $\hat{g}$. This is the main idea behind the proof of Theorem 1.1, which is given in Section 2.

Let us also observe that the first integral $H$ defined in (1.1) could be trivial (a constant), in which case our theorem does not provide any new information about the vector field $X$. The fact that this case can indeed happen (even when $X$ has non-trivial first integrals) is illustrated in Section 2, thus showing that our way of producing first integrals is by no means the most general one. Finally, in Section 3 we give some applications and examples of our main result and show how it generalizes some previous theorems in the literature.

\section{Proof of Theorem 1.1 and an example}

First we shall prove Theorem 1.1.

Proof of Theorem 1.1. Our method of proof, which is coordinate-free, makes use of standard tools from differential geometry, the reader who is not familiar with this differential geometric setting can consult e.g. [1, Chapter 2]. We first recall the following identity:

$$
L_{[X, Y]}=L_{X} L_{Y}-L_{Y} L_{X}
$$

Using the properties of the Lie derivative and assumptions (ii) and (iii) in Theorem 1.1, it is easy to check that

$$
\begin{aligned}
& L_{\lambda X}(f \Omega)=L_{X}(\lambda f \Omega)=L_{X}(\lambda) f \Omega, \\
& L_{\mu Y}(f \Omega)=L_{f \mu Y}(\Omega)=\operatorname{div}_{\Omega}(f \mu Y) \Omega=0 .
\end{aligned}
$$

We now compute $L_{[X, Y]}(f \Omega)$. On account of (2.2) and (2.3) and assumption $(i)$, the linearity of the Lie derivative implies that

$$
L_{[X, Y]}(f \Omega)=L_{\lambda X}(f \Omega)+L_{\mu Y}(f \Omega)=L_{X}(\lambda) f \Omega .
$$

On the other hand, the identity (2.1), assumption ( $i i)$ and the fact that the Lie derivative obeys the Leibniz rule allow to compute $L_{[X, Y]}(f \Omega)$ in a different way, that is:

$$
\begin{aligned}
L_{[X, Y]}(f \Omega) & =L_{X} L_{Y}(f \Omega)-L_{Y} L_{X}(f \Omega)=L_{X}\left[\left(\frac{L_{Y}(f)}{f}+\operatorname{div}_{\Omega}(Y)\right) f \Omega\right] \\
& =\left[L_{X}\left(\frac{L_{Y}(f)}{f}+\operatorname{div}_{\Omega}(Y)\right)\right] f \Omega .
\end{aligned}
$$


Combining this equation with (2.4), we finally conclude that the function

$$
H:=\frac{L_{Y}(f)}{f}+\operatorname{div}_{\Omega}(Y)-\lambda
$$

satisfies the equation

$$
L_{X}(H)=0,
$$

which implies that $H$ is a first integral of the vector field $X$. Observe that $H$ is well defined (and $C^{\infty}$ ) in the set $U:=M \backslash Z_{f}$, where $Z_{f}:=\{p \in M: f(p)=0\}$. This completes the proof of the theorem.

Remark 2.1. Arguing exactly as in the proof of Theorem 1.1 it is obtained that the function

$$
g:=L_{Y} f+f \operatorname{div}_{\Omega}(Y)-\lambda f
$$

is a second integrating factor of the vector field $X$. This implies that $H:=g / f$ is a first integral of $X$, thus providing an alternative approach to the proof of Theorem 1.1 in the spirit of the explanation given at the end of Section 1: the first integral $H$ follows from the existence of an appropriately chosen second integrating factor.

We finish this section by observing that, in the case that the function $H$ is identically constant, it provides a trivial first integral of $X$. The following example shows that this situation can indeed happen, although it is not the general case as we shall see in Section 3.

Example 2.1. Let $X$ be a volume-preserving vector field having a volume-preserving centralizer $Y$ which is (almost everywhere) transverse to $X$, that is

$$
[X, Y]=0, \quad \operatorname{div}_{\Omega}(X)=\operatorname{div}_{\Omega}(Y)=0, \quad \operatorname{rank}(X, Y)=2 \quad \text { a.e. in } M .
$$

It is straightforward to check that assumptions $(i)-(i i i)$ in Theorem 1.1 are satisfied by the vector field $X$. On the other hand, the function $H$ is identically zero, thus providing a trivial first integral of $X$. When the manifold $M$ is 3 -dimensional, it can be shown $[9,15]$ that

$$
d\left(i_{X} i_{Y} \Omega\right)=0,
$$

thus concluding, if the first De Rham cohomology group of $M$ is trivial, that is $H^{1}(M ; \mathbb{R})=0$, that there exists a function $I \in C^{\infty}(M)$ such that $d I=i_{X} i_{Y} \Omega$. This function $I$ is non-trivial because the vector field $Y$ is transverse to $X$, and since $L_{X}(I)=i_{X} d I=i_{X} i_{X} i_{Y} \Omega=0$, it is a first integral of $X$. Accordingly, the vector field $X$ has a global first integral which is not produced by the mechanism involved in Theorem 1.1.

Remark 2.2. Note that we have not assumed that the vector field $Y$ be (almost everywhere) transverse to $X$ in the statement of Theorem 1.1. If $Y$ is proportional to $X$, i.e. $Y=h X$ for some function $h$, then we have that $\lambda=L_{X}(h)$, and being $\operatorname{div}_{\Omega}(X)=\frac{-L_{X}(f)}{f}$, it easily follows that $H=0$. We conclude that, in order to obtain a non-trivial first integral $H$ with Theorem 1.1, the vector field $Y$ must be transverse to $X$.

\section{Applications and examples}

A particularly useful application of Theorem 1.1 is when the vector field $Y$ is a normalizer of $X$ (a particular case of assumption $(i)$ in the theorem) and $X$ admits an integrating factor. In this case, a well known property of the normalizers of a vector field allows to obtain many first integrals (not necessarily independent). The following corollary is a generalization of a theorem in $[6,7]$ which states that $\operatorname{div}_{\Omega}(Y)$ is a first integral of $X$ provided that $[X, Y]=0$ and $\operatorname{div}_{\Omega}(X)=0$. 
Corollary 3.1. Assume that a vector field $X \in \mathfrak{X}^{\infty}(M)$ has a normalizer $Y \in \mathfrak{X}^{\infty}(M)$, i.e. $[X, Y]=\lambda X$ for some function $\lambda \in C^{\infty}(M)$. Then, if $X$ admits an integrating factor $f$, the function $H$ defined in (1.1) is a first integral of $X$. Moreover, the function $H_{k}:=$ $L_{Y}\left(L_{Y}\left(\cdots\left(L_{Y}(H)\right)\right)\right)$ is also a first integral of $X$, where the Lie derivative $L_{Y}$ is applied $k \geq 1$ times on $H$.

Proof. It is obvious that the hypotheses of Theorem 1.1 are fulfilled with $\mu=0$, and therefore the function $H$ is a first integral of $X$. If we prove that the function $H_{1}:=L_{Y}(H)$ is a first integral of $X$ it easily follows by induction that any function $H_{k}$ is also a first integral of $X$, so let us prove that this is the case for $H_{1}$. Indeed,

$$
L_{X}\left(H_{1}\right)=L_{X}\left(L_{Y}(H)\right)=L_{[X, Y]}(H)+L_{Y}\left(L_{X}(H)\right)=\lambda L_{X}(H)=0,
$$

where we have used the identity $(2.1)$ and that $L_{X}(H)=0$, thus proving the claim.

Notice that at most $n-1$ independent first integrals can be obtained with this procedure, so most of the functions $H_{k}$ will not add any new information. On the other hand, it is relevant to remark that the existence of an integrating factor for a vector field is not enough in general to guarantee the existence of a first integral, so it is necessary to assume further hypotheses, as in the statement of Corollary 3.1.

In the following corollary we give a sufficient condition in order that a vector field be completely integrable, i.e. to admit $n-1$ independent first integrals (we say that two functions are independent if they are functionally independent in an open and dense subset of $M$ ). Completely integrable vector fields were studied in [17] concerning their connection with the period function and the existence of normalizers. Let us observe that this result is quite different from a theorem recently proved by Kozlov [12]: on the one hand, we do not need to assume that the set of vector fields $\left\{X, Y_{1}, \ldots, Y_{n-2}\right\}$ generates a (solvable) Lie algebra and on the other hand the first integrals that we obtain are explicit (i.e. $\left.\operatorname{div}_{\Omega}\left(Y_{i}\right)\right)$ and there is no need of using quadratures to compute them.

Corollary 3.2. Let $X \in \mathfrak{X}^{\infty}(M)$ be a vector field whose flow preserves the volume form $\Omega$ and admits $n-2$ normalizers $Y_{1}, \ldots, Y_{n-2}$ so that $\left[X, Y_{i}\right]=c_{i} X$ with $c_{i} \in \mathbb{R}, i=1, \ldots, n-2$. Then, if the functions $\operatorname{div}_{\Omega}\left(Y_{i}\right)$ are independent, the vector field $X$ is completely integrable.

Proof. By assumption, $\operatorname{div}_{\Omega}(X)=0$, and hence Corollary 3.1 can be applied with $f=1$ and $\lambda=c_{i}$ to imply the existence of $n-2$ first integrals $H_{i}=\operatorname{div}_{\Omega}\left(Y_{i}\right)$. Under the assumption that the functions $H_{i}$ are independent, it is standard that the pull-back of the vector field $X$, which is volume-preserving, to the regular level sets (of dimension 2 ) of the map $\left(H_{1}, \ldots, H_{n-2}\right)$ : $M \rightarrow \mathbb{R}^{n-2}$ admits an integrating factor with respect to the induced volume form, and hence it is integrable [8, Chapter 2.11]. This defines a first integral $H_{n-1}$ in any contractible domain of the regular level sets, thus proving the claim.

Remark 3.1. According to Corollary 3.2, the vector field $X$ has $n-2$ first integrals given by $H_{i}=\operatorname{div}_{\Omega}\left(Y_{i}\right)(i=1, \ldots, n-2)$, and hence we can apply [2, Chapter 4.4, Theorem 13] to conclude that on each regular level set of the first integrals the field can be integrated by quadratures. Moreover, assuming that the regular level set is connected and compact and $X$ is non-vanishing, it follows that it is diffeomorphic to a torus and $X$ is orbitally conjugated to a linear field of (rational or irrational) frequency on the level set. Observe that, in order to use [2, Chapter 4.4, Theorem 13], it is necessary to have $n-2$ first integrals, and Corollary 3.2 precisely gives a sufficient condition for this.

Theorem 1.1 can be applied to compute first integrals of Hamiltonian vector fields with symmetries (note that a Hamiltonian vector field is divergence-free with respect to some volume 
form $\Omega$ ). In the following example, which is inspired by a paper of Hojman [10], we apply it to the motion of a particle in a spherically symmetric homogeneous potential to obtain the well known conservation laws of the energy and the angular momentum. We find this result rather surprising, as the method of proof is completely different from the classical one, thus showing the existence of a mechanism different from Noether's theorem to produce first integrals in Hamiltonian mechanics.

Example 3.1. Let $V(u)$ be a real valued function which is homogeneous of degree $\alpha \in \mathbb{R} \backslash\{0\}$, that is $V(u):=u^{\alpha}$. Endowing $\mathbb{R}^{2 n}$ with Cartesian coordinates $\left(x_{1}, \ldots, x_{n}, p_{1}, \ldots, p_{n}\right)$, the Hamiltonian vector field which describes the dynamics of a particle under the action of the potential $V\left(x_{1}^{2}+\cdots+x_{n}^{2}\right)$ is given by

$$
X=p_{1} \partial_{x_{1}}+\cdots+p_{n} \partial_{x_{n}}-2 x_{1} V^{\prime} \partial_{p_{1}}-\cdots-2 x_{n} V^{\prime} \partial_{p_{n}},
$$

where $V^{\prime}(u)=\alpha u^{\alpha-1}$. It is obvious that $X$ is divergence-free with respect to the standard volume form $\Omega=d x_{1} \wedge \cdots \wedge d x_{n} \wedge d p_{1} \wedge \cdots \wedge d p_{n}$, that is $\operatorname{div}_{\Omega} X=0$. A straightforward computation shows that the vector field

$$
Y_{i j}:=\left(x_{i} p_{j}-x_{j} p_{i}\right)\left[\alpha^{-1}\left(x_{1} \partial_{x_{1}}+\cdots+x_{n} \partial_{x_{n}}\right)+p_{1} \partial_{p_{1}}+\cdots+p_{n} \partial_{p_{n}}\right]
$$

verifies

$$
\left[X, Y_{i j}\right]=\left(\alpha^{-1}-1\right)\left(x_{i} p_{j}-x_{j} p_{i}\right) X
$$

for all $1 \leq i<j \leq n$. Applying Theorem 1.1 with $f=1$ and $\lambda_{i j}=\left(\alpha^{-1}-1\right)\left(x_{i} p_{j}-x_{j} p_{i}\right)$ we conclude that $X$ has the following set of first integrals:

$$
H_{i j}=\operatorname{div}_{\Omega}\left(Y_{i j}\right)-\lambda_{i j}=\left(n\left(1+\alpha^{-1}\right)+2\right)\left(x_{i} p_{j}-x_{j} p_{i}\right),
$$

for all $1 \leq i<j \leq n$, which just expresses the well known conservation law of the angular momentum. Observe that the first integral $H_{i j}$ is trivial only when $\alpha=\frac{-n}{n+2}$. Analogously, if we consider the vector field

$$
\begin{aligned}
Y:= & \left(\frac{1}{2}\left(p_{1}^{2}+\cdots+p_{n}^{2}\right)+V\left(x_{1}^{2}+\cdots+x_{n}^{2}\right)\right) \\
& \times\left[\alpha^{-1}\left(x_{1} \partial_{x_{1}}+\cdots+x_{n} \partial_{x_{n}}\right)+p_{1} \partial_{p_{1}}+\cdots+p_{n} \partial_{p_{n}}\right]
\end{aligned}
$$

we get that

$$
[X, Y]=\left(\alpha^{-1}-1\right)\left(\frac{1}{2}\left(p_{1}^{2}+\cdots+p_{n}^{2}\right)+V\left(x_{1}^{2}+\cdots+x_{n}^{2}\right)\right) X
$$

and hence Theorem 1.1 implies that $X$ has the following first integral as well:

$$
H=\left(n\left(1+\alpha^{-1}\right)+3-\alpha^{-1}\right)\left(\frac{1}{2}\left(p_{1}^{2}+\cdots+p_{n}^{2}\right)+V\left(x_{1}^{2}+\cdots+x_{n}^{2}\right)\right) .
$$

This first integral reflects the conservation of the energy, and is non-trivial unless $\alpha=\frac{1-n}{n+3}$. Note that this example includes the $n$-dimensional harmonic oscillator (with $\alpha=1$ ) and the $n$-dimensional Kepler problem (with $\alpha=(2-n) / 2$ ) for $n>2$.

We finish this letter by elaborating on the 2-dimensional case. This is a rather exceptional situation because the existence of a normalizer implies the existence of an integrating factor, and vice versa, and therefore Theorem 1.1 can be applied. In Example 3.2 below we show that the first integral $H$ in (1.1) turns out to be trivial for the usual choice of integrating factor (normalizer) constructed from a given normalizer (an integrating factor). Nevertheless, the first integral $H$ provided by Theorem 1.1 is not trivial in general for 2-dimensional vector fields, as shown in Example 3.3. 
Example 3.2. Let $X$ be a vector field on a 2-dimensional manifold $M$ which admits a transverse normalizer $Y$, i.e. $[X, Y]=\lambda X$ and $\operatorname{rank}(X, Y)=2$ a.e. in $M$. It is immediate to check [5] that the function $f:=\left(i_{X} i_{Y} \Omega\right)^{-1}$ is an integrating factor of $X$. We now compute the function $\lambda$ in terms of $Y$ and $f$. Defining the vector field $W:=f X$ and the 1 -form $\omega:=i_{W} \Omega$, it follows that $i_{W} i_{Y} \Omega=1$ and using Cartan's formula for the Lie derivative we get

$$
L_{W}\left(i_{Y} \Omega\right)=i_{W}\left(d_{Y} \Omega\right)+d\left(i_{W} i_{Y} \Omega\right)=\operatorname{div}_{\Omega}(Y) \omega .
$$

On the other hand, since the identity $L_{W} i_{Y}=i_{[W, Y]}+i_{Y} L_{W}$ holds, we derive that

$$
L_{W}\left(i_{Y} \Omega\right)=\left(\lambda-\frac{L_{Y}(f)}{f}\right) \omega
$$

where we have used that $[W, Y]=\left(\lambda-\frac{L_{Y}(f)}{f}\right) W$ and that $L_{W}(\Omega)=0$. Identifying equations (3.1) and (3.2) and noticing that the 1-form $\omega$ is not trivial, we conclude that

$$
\lambda=\operatorname{div}_{\Omega}(Y)+\frac{L_{Y}(f)}{f} .
$$

Therefore, the first integral $H$ defined in (1.1) is identically zero for this choice of integrating factor $f$ given a normalizer $Y$.

Conversely, if $X$ admits an integrating factor $f$, it is easy to check [5] that the vector field $Y:=\left(f^{2} \operatorname{div}_{\Omega}(X)\right)^{-1} Z$ is a normalizer of $X$, with $Z$ the unique vector field which solves the equation $i_{Z} \Omega=-d f$. Arguing exactly as before it is straightforward to check the normalizer condition $[X, Y]=\lambda X$, where

$$
\lambda=\operatorname{div}_{\Omega}(Y)+\frac{L_{Y}(f)}{f}
$$

and therefore the first integral $H$ of (1.1) is also trivial for this choice of normalizer $Y$ given an integrating factor $f$.

We observe that a non-trivial first integral of $X$ can be obtained through the classical Lie's integration algorithm [16] for two dimensional vector fields with a transverse normalizer $Y$. Indeed, the 1-form

$$
\omega:=\frac{i_{X} \Omega}{i_{X} i_{Y} \Omega}
$$

is closed, and hence there exists a smooth function $I$ defined in any contractible domain of the manifold $M$ such that $d I=\omega$. It is clear that this function $I$ is a first integral of the vector field $X$.

Example 3.3. Consider the following polynomial vector field in $\mathbb{R}^{2}$ (we endow the plane with Cartesian coordinates $(x, y)$ and the standard volume form $d x \wedge d y$ ):

$$
X=\frac{1}{1+x^{2}}\left[-4 y^{3} \partial_{x}+\left(1+x^{4}+y^{4}+4 x^{3}\right) \partial_{y}\right] .
$$

It is easy to check that

$$
[X, Y]=\lambda X
$$

where the vector field $Y$ is given by

$$
Y=\frac{\left(1+x^{4}+y^{4}\right)^{2} e^{x}}{\left(1+x^{4}+y^{4}+4 x^{3}\right)^{2}+16 y^{6}}\left[\left(1+x^{4}+y^{4}+4 x^{3}\right) \partial_{x}+4 y^{3} \partial_{y}\right],
$$


and the expression of the function $\lambda$ is omitted for the sake of simplicity. Moreover, the function

$$
f=\left(1+x^{2}\right) e^{x}
$$

is an integrating factor of the vector field $X$.

Therefore, we can apply Corollary 3.1 to conclude that the function

$$
H=\frac{L_{Y}(f)}{f}+\operatorname{div}(Y)-\lambda=2\left(1+x^{4}+y^{4}\right) e^{x}
$$

is a non-trivial global first integral of $X$.

For the sake of completeness, we finally compute the first integral $I(x, y)$ of $X$ obtained through the classical Lie's integration algorithm (see the last paragraph of Example 3.2). After a few computations we get that

$$
I(x, y)=\int \frac{4 y^{3} e^{-x}}{\left(1+x^{4}+y^{4}\right)^{2}} d y=-\frac{e^{-x}}{1+x^{4}+y^{4}}=-2 H^{-1}
$$

thus showing that, in general, our approach gives a first integral different from the one obtained through classical methods, although of course in the two-dimensional case both first integrals are functionally dependent.

\section{Acknowledgements}

The authors acknowledge three referees for corrections and comments which have helped to improve the presentation of this paper. J.Ll. is partially supported by a MICINN/FEDER grant no. MTM2008-03437, by an AGAUR grant no. 2009SGR-410 and by ICREA Academia. D.P.-S. is supported by a MICINN grant no. MTM2010-21186-C02-01, by the ICMAT Severo Ochoa grant no. SEV-2011-0087 and by the Ramón y Cajal program.

\section{References}

[1] Abraham R., Marsden J.E., Foundations of mechanics, 2nd ed., Benjamin/Cummings Publishing Co. Inc. Advanced Book Program, Reading, Mass., 1978.

[2] Arnold V.I., Kozlov V.V., Neishtadt A.I., Mathematical aspects of classical and celestial mechanics, SpringerVerlag, Berlin, 1997.

[3] Bluman G.W., Anco S.C., Symmetry and integration methods for differential equations, Applied Mathematical Sciences, Vol. 154, Springer-Verlag, New York, 2002.

[4] Campoamor-Stursberg O.R., González-Gascón F., Peralta-Salas D., Dynamical systems embedded into Lie algebras, J. Math. Phys. 42 (2001), 5741-5752.

[5] García I.A., Grau M., A survey on the inverse integrating factor, Qual. Theory Dyn. Syst. 9 (2010), 115-166, arXiv:0903.0941.

[6] González-Gascón F., On a new first integral of certain dynamical systems, Phys. Lett. A 61 (1977), 375-376.

[7] González-Gascón F., Peralta-Salas D., Symmetries and first integrals of divergence-free $\mathbb{R}^{3}$ vector fields, Internat. J. Non-Linear Mech. 35 (2000), 589-596.

[8] Goriely A., Integrability and nonintegrability of dynamical systems, Advanced Series in Nonlinear Dynamics, Vol. 19, World Scientific Publishing Co. Inc., River Edge, NJ, 2001.

[9] Haller G., Mezić I., Reduction of three-dimensional, volume-preserving flows with symmetry, Nonlinearity 11 (1998), 319-339.

[10] Hojman S.A., A new conservation law constructed without using either Lagrangians or Hamiltonians, J. Phys. A: Math. Gen. 25 (1992), L291-L295.

[11] Huang D., A coordinate-free reduction for flows on the volume manifold, Appl. Math. Lett. 17 (2004), 17-22. 
[12] Kozlov V.V., Remarks on a Lie theorem on the integrability of differential equations in closed form, Differ. Equ. 41 (2005), 588-590.

[13] Marcelli M., Nucci M.C., Lie point symmetries and first integrals: the Kowalevski top, J. Math. Phys. 44 (2003), 2111-2132, nlin.SI/0201023.

[14] Merker J., Theory of transformation groups, by S. Lie and F. Engel (Vol. I, 1888). Modern presentation and english translation, arXiv:1003.3202.

[15] Mezić I., Wiggins S., On the integrability and perturbation of three-dimensional fluid flows with symmetry, J. Nonlinear Sci. 4 (1994), 157-194.

[16] Olver P.J., Applications of Lie groups to differential equations, Graduate Texts in Mathematics, Vol. 107, 2nd ed., Springer-Verlag, New York, 1993.

[17] Peralta-Salas D., Period function and normalizers of vector fields in $\mathbb{R}^{n}$ with $n-1$ first integrals, J. Differential Equations 244 (2008), 1287-1303.

[18] Prelle M.J., Singer M.F., Elementary first integrals of differential equations, Trans. Amer. Math. Soc. 279 (1983), 215-229.

[19] Prince G., Comment on "Period function and normalizers of vector fields in $\mathbb{R}^{n}$ with $n-1$ first integrals", J. Differential Equations 246 (2009), 3750-3753.

[20] Sherring J., Prince G., Geometric aspects of reduction of order, Trans. Amer. Math. Soc. 334 (1992), 433-453.

[21] Walcher S., Plane polynomial vector fields with prescribed invariant curves, Proc. Roy. Soc. Edinburgh Sect. A 130 (2000), 633-649. 